\title{
Concepto lego de estrés en estudiantes universitarios
}

\author{
Francisco Revuelta-Pérez \\ Universidad de Huelva \\ Francisco J. Atienza Martín \\ Unidad de Gestión Clínica \\ Virgen de África (Sevilla)
}

\author{
Luisa María Rodríguez-Fernández \\ Universidad de Huelva \\ Carmen Losada Ruiz \\ Unidad de Gestión Clínica Las Adoratrices (Huelva)
}

\section{RESUMEN}

El término estrés ha llegado a ser uno de los más utilizados en el contexto de la salud y la enfermedad. No obstante, no hay un consenso generalizado acerca de su definición por parte de los profesionales pertinentes, como tampoco por lo que respecta a los no expertos. Si es importante esta cuestión en relación con los primeros, no deja de serlo para los segundos. En general, las creencias, conceptos y teorías legas afectan a las percepciones de las personas y a las actitudes y comportamientos que de ellos se derivan. La idea que se tenga de lo que es el estrés, sus causas y sus consecuencias influyen en su posible vivencia, en sus desencadenantes probablesyen sus repercusiones potenciales. Hasta la fecha, se han realizado muy pocas investigaciones de las representaciones legas del estrés. La presente investigación intenta aportar conocimiento sobre dichas concepciones en una muestra de estudiantes, así como al modo posible de organizar y clasificar las respuestas de los participantes. Los resultados obtenidos, entre otras cosas, indican que las palabras más utilizadas para su definición han sido las de agobio, nervios y ansiedad. Asimismo, que el esquema general es el de entenderlo como una relación estímulo-respuesta, sin que se mencionen posibles variables moderadoras entre ellos. Se analizan y discuten los datos con propuestas para investigaciones futuras.

Palabras clave: estrés, concepto lego, representación lega, estudiantes universitarios, análisis de contenido.

\section{ABSTRACT}

The stress term has become one of the most used in the context of health and disease. However, there isn't a general consensus about its definition neither among the relevant professional, nor with respect to no experts. If this issue is important regarding the first, does not cease to be for the second. In general, beliefs, concepts and lay theories affect people's perceptions, attitudes and behaviors which flow from them. The idea that people have of what is stress, its causes and its consequences may influence in their possible survival, their likely triggers and their potential impact. To date, very few investigations of lay representations of stress have been made. This research, via qualitative analysis, tries to provide knowledge about these ideas in a sample of students, as well as the possible mode to analyze and sort out the answers of the participants. The derived results, among other things, indicate that the most used words for their definition has been overwhelmed, nerves and anxiety. Likewise, the general scheme is to understand stress as a stimulus-response relationship, without mentioning intervening variables. The data are analyzed and discussed with suggestions for the future research.

Keywords: stress, ley concept, lay representation, university students, content analysis. 
En la actualidad el término estrés es utilizado con frecuencia por distintos colectivos profesionales; especialmente, por aquellos que pertenecen al campo sanitario. No obstante, hace algunas décadas también se difundió entre la población en general y ha llegado a formar parte del vocabulario al uso de ésta. Como es obvio, la extensión de su empleo se debe al interés que ha despertado; entre otras razones porque se le ha relacionado con la salud y sus trastornos (Lledó-Boyer et al., 2010) y con dificultades, problemas y consecuencias en el ámbito laboral (Lee, Joo, \& Choi, 2012) aunque, como se verá más adelante, existen otros motivos. Una simple búsqueda de dicha expresión por Internet dará en breve millones de hipervínculos, muchísimos más si se pone con la grafía inglesa de stress. Un reflejo del interés suscitado por el estrés se encuentra en la gran cantidad de artículos en revistas especializadas (Hagenaars, van Minnen, \& de Rooij, 2010), de investigaciones (Andújar \& Revuelta, 2009), de libros o de capítulos específicos (Conrad, 2011; Juster \& Lupien, 2012), de textos divulgación (Revuelta \& Andújar, 2007) o de material de autoayuda (García, 2013), algunos de estos con mucho más que una dudosa evidencia empírica y validez.

Sin embargo, a pesar de toda la atención que ha recibido el estrés, existe un problema importante en cuanto a lo que se entiende por él tanto en el ámbito académico y/o profesional como en el popular. En el primero, se suele hablar que sus definiciones responden a tres tipos de modelos: de respuesta, de estímulo e interaccionista; si bien, en la actualidad el más aceptado es el último, del que se declara que integra a los dos anteriores, aparte de po- seer sus peculiaridades específicas. Pero con esto no se han resuelto los problemas de su conceptualización. Así, por ejemplo, Kinman y Jones (2005), considerando que el estrés es el producto de un desequilibrio entre las evaluaciones de las demandas ambientales y los recursos personales, plantean que, operacionalmente, no suele apreciarse una distinción clara entre los estresores -i.e., demandas o estímulos sobre el organismo-y las tensiones -es decir, las respuestas a esas demandas-.

El concepto de estrés, como señalan Lewig y Dollard (2001), es polifacético y, por ello, muchos plantean que todavía no se ha definido de manera adecuada. Incluso hay quienes sostienen que es primordial una reevaluación del estrés y de la teoría del afrontamiento, más que seguir con su desarrollo. Así, Britner, Harris y Daniels (2004) cuestionan los principios de las teorías del estrés desde dos perspectivas: (a) contrastando los datos de historias procedentes de terapias con las ideas simplistas -así las denominan- de la teoría del estrés y del afrontamiento, y (b) realizando un examen crítico en conjunto de ciertas suposiciones, como es el de si los estresores estuvieran por ahí en el ambiente y los individuos fueran a través de un proceso de evaluación primaria y secundaria cuando tratan con los aspectos potencialmente dañinos de dicho ambiente. Para estos autores, las formulaciones que se realizan sobre el estrés y el afrontamiento tienen un valor muy reducido y proponen como fundamental, ante estas graves limitaciones, un replanteamiento en este campo.

En este punto podría formularse la pregunta siguiente: ¿por qué habiendo todas estas crí- 
ticas es posible la gran difusión del término y el gran número de investigaciones y publicaciones relacionadas con el estrés? Kinman y Jones (2005) sugieren que precisamente esa falta de especificidad de su concepto puede constituir un atractivo, ya que cabe la oportunidad de adoptar diversas definiciones y albergar bajo su cabecera potenciales estresores, tensiones y variables intervinientes. Por su parte, Barley y Knight (1992) en cuanto al crecimiento de la popularidad del estrés entre el público en general, plantean que es atribuible a su valor explicativo de amplia base, pudiendo dar cuenta de una extensa variedad de factores ambientales negativos, estados sentimentales, sensaciones físicas y cogniciones.

Barley y Knight, admitiendo que el enfoque tradicional del estrés como un fenómeno físico ha sido muy útil para el diagnóstico y tratamiento de los síntomas relacionados con él, exponen que es poco efectivo para explicar el aumento tan destacado de su uso en la sociedad moderna o la distribución social de las quejas del estrés. Si bien la comunidad científica, habitualmente, han interpretado estas dificultades como evidencia de la necesidad de mejorar modelos, medidas y diseños de investigación, estiman que habría que tomar al estrés como un fenómeno tan cultural como psicofísico, proponiendo una teoría cultural del estrés, en la cual este funcionaría como un símbolo cultural.

Si se contempla lo anterior, es fácil percibir que se dan repercusiones humanas, económicas y sociales en base al concepto de estrés que tengan las personas no expertas, en cuanto a la evaluación de los acontecimientos, va- lor etiológico del estrés, a padecimientos, búsqueda de ayuda profesional, intervenciones, expectativas, costes sanitarios, etc. (Furnham, 1997; Ro et al., 2010). Las creencias de un individuo pueden afectar a sus percepciones $y$, por tanto, a las acciones que desarrolle (Kinman \& Jones, 2005). Aquí podría aplicarse el llamado teorema de Thomas, descrito en 1928: «si los hombres definen las situaciones como reales, son reales en sus consecuencias». Por ello, es conveniente el conocimiento de las creencias, conceptos y teorías legas sobre muchas cuestiones (Furnham, 1988; Henderson, 2010; Pelechano, 2010; Sawamura et al., 2012; Schwartz et al., 2012), como es el caso del estrés; si bien, es procedente indicar que lo lego y lo científico no tienen por qué ser obligadamente diferentes -la divulgación científica puede tener sus efectos sobre creencias y comportamientos de los no expertos y ser mutuamente reforzantes (Mulhall, 1996; Pollock, 1988)-. En concreto, Parker, Finkel e Indice (1993) encontraron que los legos y los profesionales compartían creencias similares y bastante estables en cuanto a las relaciones entre el estrés y problemas de salud particulares. De todas formas, esto no es siempre así, por lo que conocer las diferencias en el uso del lenguaje, como afirma Clark (2003), facilita la sintonía con los pacientes a la hora de entender la descripción de sus dificultades y problemas, lo que puede favorecer el proceso terapéutico (Keys, Kaiser, Kohrt, Khoury, \& Brewster, 2012) y podría disminuir los llamados encuentros difíciles (Atienza, Rodríguez-Fernández, Revuelta-Pérez, Losada, \& Marín, 2012). Habría que añadir, también, que lo lego no ha de ser necesariamente simple $y$, 
lo experto, complejo (Pelechano \& GonzálezLeandro, 2004).

En la actualidad, la evidencia sugiere que el estrés es un importante constructo lego para explicar muchas enfermedades y dolencias, especialmente a nivel etiológico, desde la fatiga (Aaronson, Pallikkathayil, \& Crighton, 2003), presuntos síntomas menopáusicos (Conboy, Domar, \& O'Connell, 2001), esquizofrenia (Broussard, Goulding, Talley, \& Compton, 2010) a la hipertensión (Marshall, Wolfe, \& McKevitt, 2012; Parker, Finkel, \& Indice, 1993) y la enfermedad coronaria (French, Marteau, Senior, \& Weinman, 2002), entre otras.

Por otro lado, Rydstedt, Devereux y Furnham (2004) hallaron relaciones significativas entre las creencias legas del estrés laboral en la línea base y la tensión mental percibida, al cabo de catorce meses, utilizando para ello un cuestionario. En este estudio se centraron en las causas y alivios percibidos del estrés.

Mostrado lo anterior, cabría hacer la pregunta acerca de si el concepto lego de estrés es tan diverso como el profesional o no. Para esta cuestión la repuesta parece ser afirmativa. La variedad conceptual entre los expertos también aparece en las representaciones legas (Helman, 1985; Pollock, 1988). Sin embargo, hasta el presente se han llevado a cabo muy pocas investigaciones sobre este asunto, a pesar del gran uso del vocablo en muy distintas circunstancias y casi como un comodín explicativo de problemas o sufrimientos. Eso no es óbice para que se generen algunas inferencias de los pocos trabajos existentes. Ogden (2007), por ejemplo, en su libro sobre Psicología de la salud, dice que los no expertos lo definen en términos como presión, tensión, fuerzas extrañas desagradables o una respuesta emocional, pero no ofrece ninguna referencia formal. Por otro lado, Pollock (1988) manifiesta que, en el caso del estrés, existen muchas similitudes entre expertos y los que no lo son, dado que su difusión ha corrido paralela a su desarrollo teórico y sostiene que se trata de un concepto manufacturado que ha llegado a ser un hecho social, con un efecto marcado y directo sobre las creencias legas y los comportamientos.

Como se ha comentado, existen pocos trabajos empíricos especíícos; entre éstos se encuentran los de Clark (2003), Helman (1985), Jex, Beehr y Roberts (1992), Kinman y Jones (2005) y Lewig y Dollard (2001). En el primero se entrevistó a 42 pacientes, unos con problemas respiratorios $\mathrm{y}$, otros, gastrointestinales. Helman encontró que las emociones y el estrés se daban como explicaciones etiológicas en un $95 \%$ de los casos pero, además, observó una cierta variedad en lo que se entendía por estrés. Estaban los que hacían referencia a respuestas del organismo, como sentirse apretado o tirante; $y$ los que se orientaban al modelo de estímulo, con mayor fijación en los conflictos externos (con la pareja, hijos, amigos o vecinos). Incluso hubo quien argumentó que tenía una tendencia genética que le colocaba el estrés psicológico dentro de su cuerpo.

Posteriormente, Jex, Beehr y Roberts (1992) citan cómo los dos últimos autores persiguieron la detección de lo que había de común en el uso de la palabra estrés entre los investigadores, buscando manualmente entre los artículos de seis revistas importantes en el comportamiento organizacional. Cada artículo en 
los que aparecía dicho término o el de estresante se asignó a una de las siguientes categorías: estímulo, respuesta, estímulo-respuesta y empleo no claro. Los desacuerdos fueron clasificados por consenso. De los 51 hallados, 21 (41\%) utilizaban alguna de esas palabras para referirse a condiciones de estímulo; 11 (22\%), a respuesta; 13 (25\%) a condiciones estímulorespuesta; y 7 (14\%) a los no claros. Según informaron, aunque hubo una leve tendencia de los investigadores para la definición como estímulo, existía una considerable variación.

Otro de los trabajos es el de Lewig y Dollard (2001) en el que se estudia lo que se vertía en los medios escritos australianos con respecto al estrés, en este caso, laboral. Seleccionaron para ello rotativos importantes de aquel país, durante el periodo de un año. Los artículos se examinaron de acuerdo con las ideologías y temas dominantes y se dio atención al lenguaje utilizado para describirlo, el público objetivo y las voces representadas en ellos. Los resultados mostraron que el estrés laboral se representaba como una epidemia económicamente costosa, una consecuencia de condiciones laborales desfavorables pero con remedios individuales y situados preferentemente en el sector público. Se aprecia, pues, sobre todo el enfoque de estímulo y una perspectiva no mecanicista.

Por su parte, Clark (2003) entrevistó a un total de 14 pacientes que habían sufrido un infarto de miocardio (IM), a las 48 horas de producido, a la semana, al mes y a los tres meses. Al final tuvo 44 entrevistas con las que efectuó un análisis cualitativo. Aunque le interesaban, fundamentalmente, las relaciones de las creencias legas del estrés con la citada patología, obtuvo algunas conclusiones en cuanto al concepto del mismo, siendo este considerado como: (a) una parte de la vida cotidiana, (b) una causa del IM, (c) una norma social, (d) un rasgo personal, (e) una respuesta a los acontecimientos vitales mayores, $\mathrm{y}(\mathrm{f})$ una respuesta a los acontecimientos diarios.

En el primero de los grupos, el estrés como parte de la vida cotidiana, se encuadraban las respuestas que lo asociaban con la vida diaria, sin que se considerara como una consecuencia del IM. En el segundo, como causa del infarto de miocardio, el estrés se vinculó con una vida ocupada o con exigencias, asociadas al trabajo, con circunstancias angustiantes y con problemas en las relaciones, que incidían a nivel orgánico. En el tercero, como una norma social, el estrés cotidiano se veía procediendo de una sociedad más amplia, con un estilo de vida apresurado. En el cuarto, como un rasgo personal, el estrés se fundaba en la predisposición del individuo ante determinadas situaciones. En el quinto, como una respuesta a los acontecimientos vitales mayores, se identificaba con situaciones particulares (p. ej., el divorcio). Y, en el sexto, una respuesta a los acontecimientos diarios, se asoció con lo que sucedía en el día en las vidas (p. ej., relaciones familiares problemáticas). Puede percibirse una correspondencia con los modelos de estímulo y de respuesta.

Debido a la muestra tan pequeña los resultados no son generalizables, si bien, están en cierto modo en consonancia con lo que se recoge en la escasa literatura, con un cierto solapamiento de las perspectivas legas y profesionales. De todas formas, los grupos realiza- 
dos tienen el inconveniente de no ser del todo excluyentes. Por otro lado, hay que tener presente que a dichos pacientes se les proporcionaron folletos sobre el infarto de miocardio y los factores de riesgo, previamente a las entrevistas, que pudieron mediatizar las respuestas.

Centrándose en el estrés laboral, Kinman y Jones (2005) realizaron un análisis de contenido de las respuestas a una entrevista semiestructurada con 45 individuos de distintos trabajos. Encontraron similitudes y diferencias entre los discursos legos y profesionales, concluyendo que las representaciones del estrés laboral eran polifacéticas, con un amplio rango de factores personales, ambientales y sociales, considerando que sus hallazgos presentaban un rango diferente y más complejo en la definiciones de estrés laboral y de la manera en cómo impactaba en relación con estudios anteriores.

En conclusión, el concepto lego de estrés está todavía muy inexplorado y, sin embargo, como se ha expuesto tiene especial importancia no sólo por lo meramente relativo a su conocimiento sino por sus repercusiones humanas, sociales y económicas. Dentro de una línea de investigación más general en el campo de las creencias, conceptos y teorías legas, el presente estudio tiene como objetivos averiguar, en una muestra de estudiantes universitarios, cuáles son los términos más empleados para definir el concepto de estrés a nivel lego, verificar si las definiciones de los no expertos pueden agruparse bajo los modelos de respuesta, estímulo e interaccionista y tratar de encontrar un esquema básico de la representación lega del estrés.

\section{Método}

\section{Muestra}

En un principio la muestra estuvo formada por un total de 140 estudiantes universitarios de primer curso. De ellos, tres no respondieron a la pregunta efectuada y uno ofreció una respuesta inclasificable, por lo que los análisis se han llevado a cabo con 136 sujetos, de los cuales 37 (27,2\%) eran varones y 99 (72,8\%), mujeres. La edad osciló entre los 17 y los 52 años, con una media de 20,03 ( $D E=4,061)$ y una mediana de 19 y una moda de 18. No hubo ningún criterio de exclusión.

\section{Procedimiento}

A los sujetos, en el aula, se les invitó nada más comenzar el curso académico a participar voluntariamente en este trabajo. No se produjo ninguna oposición a responder a las preguntas que se les formularan. No obstante, antes se hizo una lectura pública de los objetivos de la investigación así como del anonimato que se mantendría sobre los participantes, enfatizando el hecho de que ningún dato facilitaría o daría a conocer la identidad de cualquiera de ellos. Se les comunicó, además, que los resultados se emplearían dentro de la temática de la docencia.

\section{Cuestionario}

Dadas las características del estudio no se elaboró un cuestionario extenso. Los individuos tan solo tenían que responder a la pregunta siguiente: De forma concisa, díganos lo que significa para usted estrés o estar estresado. Se trata de que exponga lo que cree que verdaderamente es, al margen de que coincida o no 
con lo que le hayan dicho, leído o escuchado por cualquier medio. Las respuestas se registraron por escrito por parte de los propios participantes.

\section{Análisis de las respuestas}

Una vez agrupadas las respuestas de cada sujeto a la pregunta citada se procedió a realizar un conteo de palabras clave o de sus derivaciones lingüísticas en las definiciones ofrecidas para la identificación de las más mencionadas. Aunque de carácter arbitrario, se estimó procedente, teniendo en cuenta el número total de la muestra, tomar como criterio todas aquellas que había sido utilizadas diez o más veces. Asimismo, mediante la herramienta contar palabras de un programa de tratamiento de texto se registraron el número de palabras, el de caracteres sin espacios y el de los mismos con espacios, con el propósito de establecer comparaciones con otras muestras diferentes en un futuro.

A continuación se llevó a cabo un análisis de contenido de las definiciones, siguiendo las recomendaciones de Polit y Hungler (1991/1994) y de Sommer y Sommer (1997/2001).

Después de varias revisiones se optó por establecer en primer lugar cuatro categorías, relacionadas con los agrupamientos que se hacen en la literatura académica sobre el concepto de estrés. La primera de ellas era estímulo. En este caso, se registraban aquellas respuestas que sólo aludían en la definición estímulos para indicar lo que era el estrés. La segunda, respuesta, similar a lo anterior, pero aquí sólo si se hacía mención a respuestas. La tercera estímulo-respuesta, si el sujeto indicaba las dos anteriores. Y, la cuarta, no mecanicista. Con esta última se pretendía averiguar si la persona entendía el estrés como algo más allá de lo meramente mecánico y reflejaba algo que tuviera que ver con un proceso, transacción o interacción. Teniendo en cuenta que en más de una redacción se dieron problemas de interpretación en este aspecto se adoptó el criterio de que el respondedor sugiriera una cierta relatividad en la respuesta de estrés en base al proceso de evaluación cognitiva; esto es, algún signo de superación de un punto de vista mecanicista del estrés. Algunos ejemplos de contestaciones de estas características pueden ser: «El estrés depende de las características personales de cada uno», «El estrés depende de la forma que tiene esa persona de realizar sus actos y en su manera de pensar y vivir la vida» 0 «El estrés se pasa intentando enfocar el problema de forma positiva».

Posteriormente, se llevó a cabo un segundo análisis de contenido pero, en este caso, con los estímulos, por un lado, y con las respuestas, por otro. Con el fin de no caer en errores o, más bien, en interpretaciones inadecuadas o fallidas se decidió simplificar al máximo las categorizaciones. Con este intención, para estímulo se formaron tres grupos: a) situaciones o estímulos negativos, adversos o que presionan; b) factor tiempo, si el sujeto resaltaba que esta variable jugaba un papel en el desencadenamiento del estrés; $y, c)$ estilo de vida, cuando se hacía referencia al mismo (ver Tabla 1).

Con respecto a respuesta, los grupos fueron cinco: a) activación, alteración, saturación y similares: aquí se han recogido todas aquellas contestaciones en las que se ha entendido el 
estrés como algo que activa al sujeto; b) desactivación, bloqueos e incapacidades, lo contrario de lo anterior, pero añadiendo el hecho de que puede generar pérdida de capacidad personal en cualquier sentido; c) repercusiones sobre la salud y síntomas físicos, cuando se ha considerado que la respuesta de estrés origina síntomas físicos o que afecta, en general y de manera negativa, a la salud; d) malestar e incomodidad, expresado de una manera más bien global; y e) explicaciones psicológicas, si se ha expresado algo relativo a los aspectos psicológicos en la respuesta (ver Tabla 2 ).

\section{Resultados}

El término más mencionado, con sus derivaciones lingüísticas, fue agobio, que tuvo 57 citaciones, correspondientes a 49 sujetos, ya que uno lo empleó tres veces en su definición $y$, seis de ellos, dos veces. Le siguieron nervios (35), ansiedad (25), malestar (20), tensión (13), acumulación (12) y presión (11). Con estos últimos no se produjeron repeticiones a la hora de definir el estrés (ver Tabla 3).

En relación con el número de palabras la media se ha situado en $35,04(D E=21,01)$, osci- lando desde 7 a 221 y una moda de 25 . Con los caracteres sin espacio ha sido de 164,46 (DE = $69,52)$, yendo desde 33 a 414 y con una moda de 106. Y, con los caracteres con espacio, de $196,19(D E=81,85)$, con un mínimo de 39 y un máximo de 494 y una moda de 154. Entre varones y mujeres no hubo diferencias estadísticamente significativas en el número de palabras utilizadas, pero sí en el número de caracteres tanto sin espacios $(t=2,151, p=0,033)$ como con espacios $(t=2,125, p=0,035)$.

En cuanto al primer nivel de categorización los resultados son los siguientes: únicamente 4 sujetos, el 2,9\%, indicaron sólo estímulos, 22 $(16,2 \%)$, exclusivamente respuestas, $y$, las dos cosas, 110 (80,9\%). En cuanto a la consideración de un sustrato no determinista, expresiones o frases que pudieran indicar una cierta relatividad en la respuesta de estrés, con una impronta no mecanicista que diera a entender la participación variables personales, entre otras, se percibió en 13 participantes, el 9,6\%.

Por lo que respecta al tipo de estímulos indicados, fue básicamente el que hace referencia a los que activan o presionan (112, 82,4\%), aunque un grupo no demasiado pequeño (35,

Tabla 1

Ejemplos de descripciones utilizadas como estímulos, según distintas categorías para definir el estrés.

\begin{tabular}{ll}
\hline \multicolumn{1}{c}{ Categorías de estímulos } & \multicolumn{1}{c}{ Ejemplos } \\
\hline Situaciones o estímulos negativos, adversos o & «Tener muchas actividades que hacer» \\
que presionan & «Aparece cuando tienes muchos problemas» \\
& «Provocado por la sucesión de distintas \\
Factor tiempo & situaciones no agradables para un sujeto» \\
& «Por no tener tiempo suficiente» \\
& «Aparece cuando disponemos de poco tiempo \\
& para desempeñar una tarea» \\
Estilo de vida & «Por la aproximación de una fecha» \\
& «Causado por sus hábitos de vida» \\
\hline
\end{tabular}


Tabla 2

Ejemplos de descripciones utilizadas como respuestas, según distintas categorías para definir el estrés

\begin{tabular}{|c|c|}
\hline Categorías de respuestas & Ejemplos \\
\hline Activación, alteración, saturación y similares & $\begin{array}{l}\text { «Estado de tensión o alerta» } \\
\text { «Sensación de agobio» } \\
\text { «Estado de nerviosismo» }\end{array}$ \\
\hline Desactivación, bloqueos e incapacidades & $\begin{array}{l}\text { «Estar en un estado de ánimo depresivo o bajo» } \\
\text { «Nos hace sentir incómodos» } \\
\text { «No eres capaz de nada» }\end{array}$ \\
\hline Repercusiones para la salud y síntomas físicos & $\begin{array}{l}\text { «Afecta fisiológicamente» } \\
\text { «Provoca graves consecuencias para la salud» } \\
\text { «Con síntomas como opresión en el pecho, } \\
\text { dolor de cabeza, taquicardias...» }\end{array}$ \\
\hline Malestar e incomodidad & $\begin{array}{l}\text { «Estado de malestar interior» } \\
\text { «El estrés es un estado de ánimo en el que la } \\
\text { persona se encuentra incómoda» } \\
\text { «Definiríamos el estrés como una sensación de } \\
\text { malestar mental» }\end{array}$ \\
\hline Explicaciones psicológicas & $\begin{array}{l}\text { «Tener un gran problema psíquico» } \\
\text { «Estado de desorden mental» } \\
\text { «Estado mental desconcertado no estable» }\end{array}$ \\
\hline
\end{tabular}

$25,7 \%$ ) estimaba como factor relevante en esa activación o presión las fechas o el hecho temporal. El estilo de vida, algo que podría parecer más relevante, fue citado nada más que por 2 personas $(1,5 \%)$.
Por su parte, lo que se refiere a la clase de respuestas, la frecuencia más elevada fue para el primer grupo de la categorización, aquellas que suponen una activación o alteración de carácter agobiante, nervioso, ansioso o similar

Tabla 3

Términos y sus derivaciones lingüísticas más citados, por encima de diez veces, y número de participantes que los mencionaron en sus definiciones de estrés

\begin{tabular}{lccc}
\hline & Término & Citaciones & \\
\cline { 1 - 2 } Agobio & 57 & 35 & $49(36,03 \%)$ \\
Nervios & 25 & $35(25,74 \%)$ \\
Ansiedad & 20 & & $25(18,38 \%)$ \\
Malestar & 13 & & $20(14,71 \%)$ \\
Tensión & 12 & $13(9,56 \%)$ \\
Acumulación & 11 & $12(8,52 \%)$ \\
Presión & & & $11(8,09 \%)$ \\
\hline
\end{tabular}


(100; 73,5\%), seguido por lo contrario pero a distancia. En concreto, el segundo grupo, de desactivación, bloqueos o incapacidades, englobó a 34 sujetos (25,0\%). A continuación, la afectación a la salud y los síntomas físicos (32; 23,5\%), los síntomas y fenómenos psicológicos $(19 ; 14,0 \%)$ y el malestar e incomodidad (17; 12,5\%) (ver Figura 1).

Para averiguar si el sexo era o no independiente en las distintas categorías se utilizó la prueba de chi cuadrado. No obstante, la distribución de frecuencias ha sido muy desigual y las observaciones en algunos grupos muy bajas, con valores incluso de 0 y 2 . Por ello, no se presentan los valores obtenidos, aunque se ofrece la información de que en todos los casos no se rechaza la hipótesis de independencia.

Por tanto, el modelo mayoritario en los participantes es el establecido por uno o varios estímulos que producen tal o tales respuestas en el organismo. Los estímulos suelen ser aquellos relacionados con las situaciones negativas, adversas o que ejercen presión sobre la persona (p. ej., tener muchas cosas que hacer, situación problemática...) y las respuestas las relativas a la activación o alteración (p. ej., ansiedad, nerviosismo...).

\section{Discusión}

El conocimiento de conceptos y teorías legas es un campo importante por cuanto tienen un papel relevante en muchas áreas y en comportamientos. Muchas decisiones, por ejemplo, están muy determinadas por las creencias que tienen las personas, las cuales no siempre se ajustan a lo que es más objetivo o a lo que los resultados científicos vienen indicando. Parece evidente, a partir de aquí, que es muy

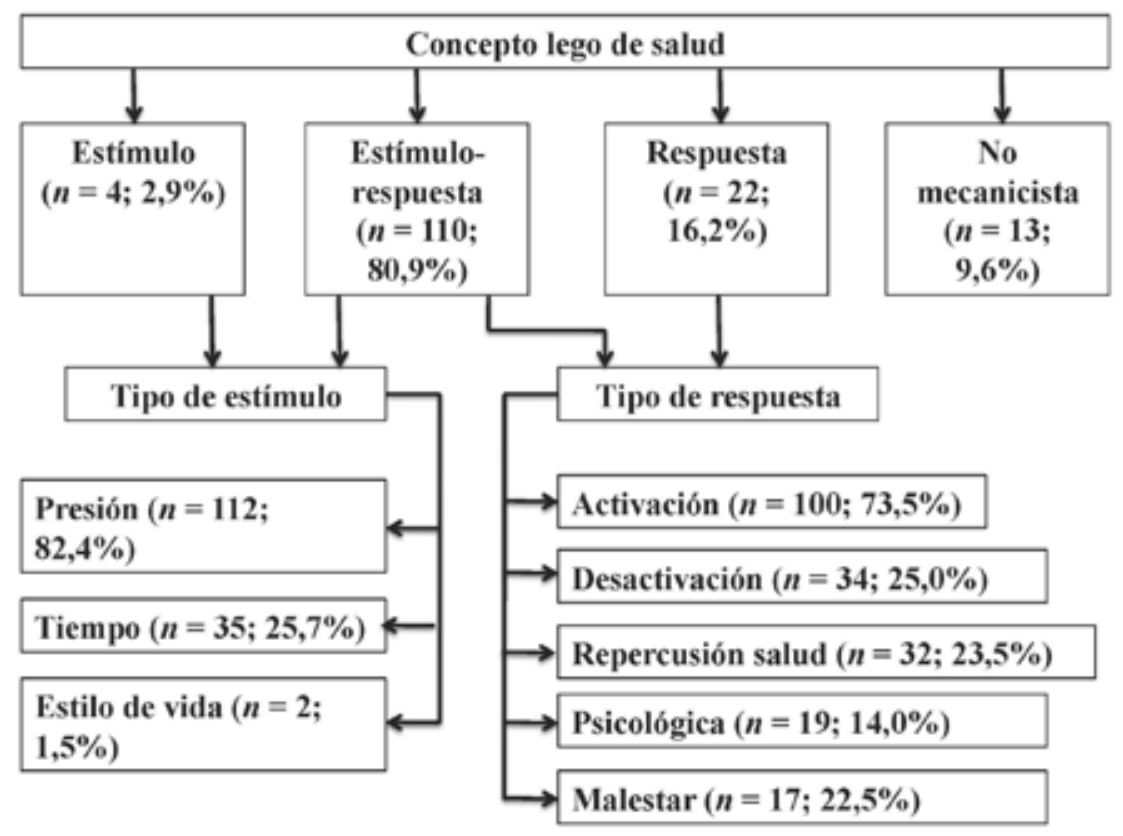

Figura 1. Frecuencias de modelos legos de estrés y de categorías de estímulos y respuestas.

Nota. Por razones de espacio se han simplificado las categorías, salvo no mecanicista y estilo de vida. Presión = situaciones u otros estímulos negativos, adversos o que presionan; Tiempo = factor tiempo; Activación = activación, alteración, saturación y similares; Desactivación = desactivación, bloqueos e incapacidades; Repercusiones salud = repercusiones sobre la salud y síntomas físicos; Psicológica = explicaciones psicológicas; y Malestar = malestar e incomodidad. 
conveniente y necesario, en bastantes ocasiones, saber cuáles son las ideas, los conceptos y las teorías elaboradas por las personas legas en materias como la salud y la enfermedad y en todo lo relacionado con ellas. Cabe, por tanto, afirmar que parece procedente averiguar lo concerniente a las representaciones legas sobre el estrés, término muy difundido y empleado, no sólo entre académicos y profesionales sino, asimismo, entre los no expertos o legos y este ha sido el objetivo fundamental del presente trabajo.

En primer lugar, los resultados indican que los términos más utilizados y que superan en su uso un $25 \%$ o más de la muestra han sido sólo dos: agobio y nervios, siguiendo, pero por debajo de un 19\%, ansiedad, malestar, tensión, acumulación y presión. Por tanto, aunque otras expresiones señaladas por Ogden (2007) también aparecen en la presente investigación existe una diferencia en cuanto a su frecuencia de aparición pues son menos nombradas. En este asunto particular, sería deseable la comparación de estos resultados con los que se obtuvieran con personas de otras edades, niveles académicos y circunstancias. Otros autores, como Kinman y Jones (2005) han puesto algunos ejemplos de los términos utilizados por sus sujetos de la muestra pero no aportan ningún dato adicional que permita la comparación con los aquí obtenidos.

Contemplar el número de palabras en las definiciones tiene que ver, aparte de ser un posible indicador de fluidez verbal, con la mayor o menor riqueza de elementos que introduzca el sujeto. Aunque está pendiente de acabar el estudio, los primeros datos con una muestra de pacientes crónicos en curso apuntan a definiciones bastante más cortas del estrés. Habrá que comprobar si esto es debido a formación académica, pues la muestra está más compuesta por personas con más bajo nivel educativo, o por otras razones. Resulta curioso el resultado en cuanto a que no hubo diferencias estadísticamente significativas entre varones y mujeres en el número de palabras empleadas, pero sí por lo que respecta a la cantidad de caracteres, con y sin espacio. De momento, no se presenta una explicación viable; si bien, en principio, parece como si las mujeres utilizaran palabras con más sílabas.

En el presente estudio los sujetos optaron por definiciones que se encuadraban en el grupo estímulo-respuesta (78,6\%), estando a mayor distancia los que sólo ofrecieron definiciones del modelo de respuesta $(15,7 \%)$ y a mucho más los que únicamente hicieron referencia al de estímulo (2,9\%). Difieren estos resultados con personas legas de los de Jex et al. (1992) para profesionales. Dichos autores encontraron dentro del grupo de estímulo el $41 \%$ de las concepciones de estrés, del de respuesta el $22 \%$ y del de estímulo-respuesta el $25 \%$. De todas formas, lo que presentan estos autores sorprende en alguna medida puesto que ya en ese periodo iba asentándose en el terreno profesional el enfoque interaccionista (Lazarus \& Folkman, 1984/1986). Tampoco concuerda esto con la revisión de medios escritos australianos llevada a cabo por Lewig y Dollar (2001) quienes identificaron el uso del término estrés primordialmente desde el enfoque de estímulo.

Tan solo el 9,6\% dieron una visión no mecanicista del estrés. No puede asegurarse que el 
resto de los sujetos $(90,4 \%)$ tengan un perspectiva contraria. En investigaciones posteriores sería un elemento a dilucidar puesto que los determinismos son susceptibles de coadyuvar o entorpecer, según su sentido, los resultados de las intervenciones de reducción o de control del estrés.

En cuanto a la categorización de estímulos mencionados por los participantes, la mayor parte $(82,4 \%)$ los identificaban con situaciones o circunstancias que activan a la persona, que ejercen una presión sobre el organismo. Resultado que no resulta extraño a partir de un mero acercamiento a este tema. Eso sí, hubo un subgrupo $(25,7 \%)$ que adjudicaron la razón de esa activación al factor temporal; esto es, no era tanto lo que hubiera que hacer (tarea, obligación o lo que fuera) lo relevante sino el cuándo o, más bien, para cuándo. También esto está en consonancia con lo que muchas veces se difunde por los medios de comunicación o en las historias de novelas o de películas. Sin embargo, algo a lo que, a veces, se recurre para explicar el estrés, el estilo de vida, ha sido mencionado muy marginalmente $(1,47 \%)$.

Los datos obtenidos en cuanto al tipo de respuestas dadas por el organismo como proceso de estrés fueron coherentes con lo anterior; esto es, similarmente, los participantes indicaron que el estrés era una respuesta de activación o alteración de carácter agobiante, nervioso o similar $(73,5 \%)$, mientras lo contrario, la desactivación, bloqueos o incapacidades fueron sólo el 25,0\%. Sin embargo, en la consideración de la respuesta de estrés también es de destacar el que afectaba a la salud, los síntomas físicos, los síntomas y fenómenos psicológicos y el malestar o incomodidad. En las respuestas a la pregunta realizada se observa variedad pero no parece ser tanta como la manifestada por Helman (1985) en su trabajo pionero.

Para ninguna de las categorías establecidas se ha encontrado diferencias entre varones y mujeres. En principio, se desconocen trabajos que pudieran reflejar algún matiz con esta variable.

Por tanto, el modelo mayoritario en los participantes es el establecido por uno o varios estímulos que producen tal o tales respuestas en el organismo. Subyace que existe un proceso pero no se especifica si se trata de algo mecánico o no, lo que es importante tener en cuenta de cara a los programas del manejo del estrés. Los estímulos suelen ser aquellos relacionados con las situaciones negativas, adversas o que ejercen presión sobre la persona (p. ej., tener muchas cosas que hacer, situación problemática...) y las respuestas las relativas a la activación o alteración (p. ej., agobio, nerviosismo...).

Por supuesto, está el problema de la generalización de los resultados presentados. Se trata de una muestra muy concreta, estudiantes universitarios. No obstante, lo principal ha sido iniciar un camino, pues no parece haber trabajos previos, y establecer una metodología de análisis de las respuestas a la pregunta planteada. Desde esta perspectiva puede concluirse que merece la pena el esfuerzo en este área de conocimiento.

Sería procedente para futuras investigaciones comparar estos resultados con los de otras muestras, en base a distintos criterios (p. ej., 
por grupos de edad, enfermedad crónica, etc.). y ver posibles relaciones con niveles de estrés autopercibido, diferentes síntomas físicos y psicológicos, así como con la calidad de vida. Pero, además, hay algo a lo que habría que darle un especial importancia implementando la manera de articularlo en futuras investigaciones: la cuestión del no mecanicismo de la respuesta de estrés. Es conocido que cuando alguien establece una relación causa-efecto entre dos hechos -aunque no exista realmente tal vínculo causal-, en bastantes ocasiones, se aumenta la probabilidad de que tal asociación se produzca -lo que es explotado en el contexto sanitario por la industria farmacéutica-, como ocurre, por ejemplo, con muchas quejas psicosomáticas. En el proceso del estrés participa un buen número de variables y, en más de una, es posible ejercer un control en diverso grado, lo que hace que no sea del todo inevitable que si sucede $A$ habrá de sobrevenir B. Intervenir sobre este particular haciendo ver la posibilidad de atenuar o minimizar hasta donde proceda el estrés y sus consecuencias constituiría, probablemente, dentro del ámbito de las creencias legas, una aportación relevante en el campo de la salud y la enfermedad, favoreciendo la erradicación de una posición de determinismo absoluto.

\section{Referencias}

Aaronson, L. S., Pallikkathayil, L., \& Crighton, F. (2003). A qualitative investigation of fatigue among healthy working adults. Western Journal of Nursing Research, 25, 419-433.

Andújar Barroso, R. T., \& Revuelta-Pérez, F. (2009). Estrés, afrontamiento y salud en el colectivo docente: datos de un estudio exploratorio en población costarricense. En
F. D. Rubio Alcalá y L. Ma Bonilla Sandoval (Eds.), Análisis del malestar laboral en los entornos educativos costarricenses (pp. 221291). San José, Costa Rica: Promesa.

Atienza Martín, F., Rodríguez-Fernández, L. M.a , Revuelta-Pérez, F., Losada Ruiz, C., \& Marín Andrés, G. (2012). Encuentros difíciles en atención primaria: una aproximación mediante grupos focales. Análisis y Modificación de Conducta, 37, 115-126.

Barley, S. R., \& Knight, D. B. (1992). Toward a cultural theory of stress complaints. Research in Organizational Behaviour, 14, 1-48.

Briner, R. B., Harris, C., \& Daniels, K. (2004). How do work stress and coping work? Toward a fundamental theoretical reappraisal. British Journal of Guidance \& Counselling, 32, 223234.

Broussard, B., Goulding, S. M., Talley, C. L., \& Compton, M. T. (2010). Beliefs about causes of schizophrenia among urban African American community members. Psychiatric Quarterly, 81, 349-362. doi:10.1007/s11126010-9143-1

Clark, A. M. (2003). 'It's like an explosion in your life...': Lay perspectives on stress and myocardial infarction. Journal of Clinical Nursing, 12, 544-553.

Conboy, L., Domar, A., \& Connell, E. (2001). Women at mid-life: Symptoms, attitudes, and choices, an Internet based survey. Maturitas, 38, 129-136.

Conrad, C. (Ed.) (2011). Handbook of stress: Neuropsychological effects on the brain (pp. 248265). Inglaterra: Blackwell Publishing.

French, D. P., Marteau, T. M., Senior, V., \& Weinman, J. (2002). The structure of beliefs about the causes of heart attacks: A network analysis. British Journal of Health Psychology, 7, 463-479.

Furnham, A. (1997). Lay theories of work stress. Work \& Stress: An International Journal of Work, Health \& Organizations, 11, 68-78.

Furnham, A. F. (1988). Lay theories. Everyday understanding of problems in the social science. Oxford, Inglaterra: Pergamon Press.

García Castro, T. (2013). Cómo superarte con el estrés positivo: un método para que el estrés 
se convierta en tu mejor aliado. Madrid: Jorge A. Mestas. Ediciones Escolares.

Hagenaars, M. A., van Minnen, \& de Rooij, M. (2010). Cognitions in prolonged exposure therapy for posttraumatic stress disorder. International Journal of Clinical and Health Psychology, 10, 421-434.

Helman, C. G. (1985). Psyche, soma, and society: The social construction of psychosomatic disorders. Culture, Medicine and Psychiatry, 9, 1-26.

Henderson, J. (2010). Expert and lay knowledge: A sociological perspective. Nutrition \& Dietetics, 67, 4-5. doi:10.1111/j.17470080.2010.01409.x

Jex, S. M., Beehr, T. A., \& Roberts, C. K. (1992). The meaning of occupational stress ítems to survey respondents. Journal of Applied Psychology, 77, 623-628.

Juster, R. P., \& Lupien, S. J. (2012). Chronic stress and allostatic load. En K. Schenck-Gustafsson, P. R., DeCola, D. W., Pfaff, \& D. S. Pisetsky (Eds.), Handbook of clinical gender medicine (pp. 70-81). Basel: Karger AG.

Keys, H. M., Kaiser, B. N., Kohrt, B. A., Khoury, N. M. \& Brewster, A.-R. T. (2012). Idioms of distress, ethnopsychology, and the clinical encounter in Haiti's Central Plateau. Social Science \& Medicine, 75, 555-564. doi:10.1016/j.socscimed.2012.03.040

Kinman, G., \& Jones, F. (2005). Lay representations of workplace stress: What do people really mean when they say they are stressed? Work \& Stress, 19, 101-120.

Lee, J.-S., Joo, E.-J., \& Choi, K.-S. (2012). Perceived stress and self-esteem mediate the effects of work-related stress on depression. Stress and Helaht, 29, 75-81.

Lewig, K. A., \& Dollard, M. F. (2001). Social construction of work stress: Australian newsprint media portrayal of stress at work. Work \& Stress, 15, 179-190. DOI: 10.1080/02678370110066986

Lledó-Boyer, A., Pastor-Mira, M. a Á., Pons-Calatayud, N., López-Roig, S., Rodríguez-Marín, \& Bruehl, S. (2010). Control beliefs, coping and emotions: Exploring relationships to explain fibromialgia health outcomes. In- ternational Journal of Clinical and Health Psychology, 10, 459-476.

Marshall, I. J., Wolfe, C. D., \& McKevitt, C. (2012). Lay perspectives on hypertension and drug adherence: Systematic review of qualitative research. British Medical Journal, 345, e3953.

Mulhall, A. (1996). Cultural discourse and the myth of stress in nursing and medicine. International Journal of Nursing Studies, 33, 455-468.

Ogden, J. (2007). Health psychology. A textbook ( $4^{\text {th }}$ ed.). Berkshire (England): Open University Press and McGraw-Hill.

Parker, J. D. A., Finkel, M. D., \& Indice, L. C. (1993). Stress and illness: Th estructure of a belief system. Canadian Journal of Behavoural Science, 25, 193-204.

Pelechano, V. (2010). Unas notas acerca de la psicología lega y modelos asimilados. Análisis y Modificación de Conducta, 36, 1-17.

Pelechano, V., \& González-Leandro, P. (2004). La concepción lega de la sabiduría, inteligencia y envejecimiento en adolescents. Análisis y Modificación de Conducta, 30, 437-460.

Polit, D., \& Hungler, B. (1994). Investigación científica en ciencias de la salud (Trad. M. ${ }^{\mathrm{a}} \mathrm{T}$. Aguilar) (4..$^{a}$ ed. en español de la 4. ${ }^{a}$ ed. en inglés). México: Nueva Editorial Interamericana. (Trabajo original publicado en 1991).

Pollock, K. (1988). On nature of social stress: Production of a modern mythology. Social Science and Medicine, 26, 381-392.

Revuelta-Perez, F., \& Andújar, R. T. (2007). Ansiedad y estrés. En Ma J. Álava (Dir.), La Psicología que nos ayuda a vivir (pp. 679-695). Madrid: La Esfera de los Libros.

Ro, K. E. I., Tyssen, R., Hoffart, A., Sexton, H., Aasland, O. G., \& Gude, T. (2010). A threeyear cohort study of the relationships between coping, job stress and burnout after a counseling intervention for help-seeking physicians. BMC Public Health, 10, 213. doi:10.1186/1471-2458-10-213

Rydstedt, L. W., Devereux, J., \& Furnham, A. F. (2004). Are lay theories of work stress related to distress? A longitudinal study in the British workforce. Work \& Stress, 18, 245254. 
Sawamura, K., Tachimori, H., Koyama, T., Koyama, A., Naganuma, Y., Kim, Y., \& Takeshima, T. (2012). Lay diagnosis and views on causes, coping strategies, and treatment for schizophrenia. Communitiy Mental Health Journal, 48, 309-316.

Schwartz, L. A., Kazak, A. E., B. W. DeRosa, M. C. Hocking, W. L. Hobbie, \& J. P. Ginsberg (2012). The role of beliefs in the relationship between health problems and posttraumatic stress in adolescent and young adult cancer survivors. Journal of Clinical Psychology in Medical Settings, 19, 138-146. doi:10.1007/s10880-011-9264-1

Sommer, B., \& Sommer, R. (2001). La investigación del comportamiento. Una guía práctica con técnicas y herramientas (Trad. A. Fernández Cerdeño Franco). México: Oxford University Press. (Trabajo original publicado en 1997). 
